\title{
Lower cranial nerve palsy during radiotherapy for glottic cancer in a patient with Wegener's granulomatosis: An interesting case report
}

\author{
GRAZIA LAZZARI ${ }^{1}$, ALESSANDRA BRIATICO VANGOSA ${ }^{2}$, MARIA ASSUNTA DE CILLIS ${ }^{3}$, \\ GIOVANNI BUCCOLIERO ${ }^{4}$ and GIOVANNI SILVANO ${ }^{1}$ \\ Departments of ${ }^{1}$ Radiation Oncology, ${ }^{2}$ Radiology, ${ }^{3}$ Otorhinolaryngology and ${ }^{4}$ Infectious Diseases, \\ S. Giuseppe Moscati Hospital, I-74100 Taranto, Italy
}

Received August 14, 2018; Accepted October 15, 2018

DOI: $10.3892 / \mathrm{mco} .2018 .1748$

\begin{abstract}
The aim of the present study was to report an unusual case of multiple lower cranial nerve palsies in a patient with Wegener's granulomatosis (WG) during radiotherapy for glottic cancer. WG is an autoimmune disease characterized by necrotizing granulomas mainly in the upper and lower respiratory tract or kidneys; however, the involvement of cranial nerves is not uncommon. Prior to the use of cyclophosphamide (CYC) the 1-year mortality rate was $\sim 82 \%$; the introduction of rituximab (RTX) has revolutionized the course of the WG, with remission rates comparable to those of CYC and superior effectiveness in relapsing patients. Hypogammaglobulinemia and $\mathrm{B}$-cell depletion are the best known monitored side effects affecting survival due to secondary infections. Immunodepression and relapse with lower cranial nerve palsy have a negative impact on prognosis. We herein present the case of a heavily pre-treated GPA patient with secondary immunosuppression, who underwent radiotherapy for glottic cancer and developed multiple low cranial nerve palsies during treatment, which was interrupted at $60 \mathrm{~Gy}$. The possible related causes and the association between previous immunosuppressive treatments and radiotherapy were also analyzed to elucidate the cause of this complication.
\end{abstract}

\section{Introduction}

Wegener's granulomatosis (WG), recently renamed as granulomatosis with polyangiitis (GPA), is an antineutrophil cytoplasmic antibody (ANCA)-associated vasculitis (AAV), usually affecting small- and medium-sized vessels. GPA

Correspondence to: Dr Grazia Lazzari, Department of Radiation Oncology, S. Giuseppe Moscati Hospital, Str. Per Martina Franca, I-74100 Taranto, Italy

E-mail: lazzarigrazia@gmail.com

Key words: granulomatosis, vasculitis, antineutrophil cytoplasmic antibodies, glottic cancer, rituximab often involves the upper and lower airways or the kidneys, but various other systems may be affected (1), such as the central nervous system (CNS), albeit infrequently (2). Involvement of the CNS occurs in $2-8 \%$ of the patients and it is most commonly characterized by cranial neuropathy. Lower cranial nerve palsies are reported as unusual manifestations occurring in the later stages of the disease and have been associated with pachymeningitis of the skull base (3). Prior to the use of cyclophosphamide (CYC), this disease was fatal (4); however, the use of CYC and rituximab (RTX) have ameliorated prognosis, inducing a response or remission in $>80 \%$ of the patients (5). Unfortunately, these agents may induce a profound and long-lasting immunosuppression due to the depletion of mature B lymphocytes and hypogammaglobulinemia, which may explain the negativity for c-ANCA, mainly during disease relapse (6). In addition, development of secondary cancers has been described during the disease course and some reports have implicated these therapies in cancer development $(7,8)$. We herein report the case of a heavily treated WG patient who developed multiple low cranial nerve palsies during radiotherapy for metachronous glottic cancer. Due to severe hypogammaglobulinemia and profound B-cell depletion, the c-ANCA titre was not detectable. The patient experienced a fatal progressive WG flare complicated by aspiration pneumonia and intestinal perforation, and succumbed to the disease 6 months later.

\section{Case report}

In August 2011, a 60-year-old Caucasian male patient presented to S. Giuseppe Moscati Hospital (Taranto, Italy) complaining of severe dysphonia. The patient's family and psychosocial history were not relevant, while his medical history included diabetes and WG diagnosed 10 years earlier on a lobectomy specimen of the left lung. The course of the disease had been characterized by recurrent episodes of otitis, sinusitis and febrile pneumonia with hemoptysis during a long chronic course associated with ANCA titre positivity. No renal, dermal, ocular or neurological manifestations had been priorly reported. The patient had been previously treated with steroids, methotrexate, CYC and RTX, obtaining a good 
clinical benefit and c-ANCA titre control. RTX had been prescribed as off-label therapy outside clinical trials, and had been administered between March 2007 and July 2011, 1 month prior to the diagnosis of glottic cancer. Although the titre of c-ANCA was $<20 \mathrm{UR} / \mathrm{ml}$ at that time, the C-reactive protein (CRP) level was $25 \mathrm{mg} / 1$ (normal value $<8 \mathrm{mg} / \mathrm{l}$ ), while the albumin level was $3.5 \mathrm{mg} / \mathrm{l}$; therefore, the Glasgow Score for this patient was 1 . The blood tests revealed a white blood cell (WBC) count of $8,000 / \mathrm{mm}^{3}$ and lymphopenia $(18 \%$ lymphocytes). Blood urea nitrogen was normal, while the protein level was $6 \mathrm{~g} / \mathrm{ml}$, with hypogammaglobulinemia (12\%). On clinical examination, the patient had no palpable lymph nodes in the neck, and reported an inexplicable weight loss of $>5 \mathrm{~kg}$ over 3 months. On suspicion of laryngeal involvement by WG, laryngoscopy was conducted and revealed a right vocal fold palsy and bilateral glottic thickening with oedema and nodular spots in the mucosal surface, mainly on the right vocal fold. The biopsy revealed infiltrating G2 squamous cell carcinoma (SCC) of the glottis, mainly involving the right false vocal fold, the ventricle and the infraglottic space. The magnetic resonance imaging (MRI) on T1 and T1 STIR sequences confirmed enlargement of the right vocal padded fold and glottic space reduction, without metastases to the neck lymph nodes (Fig. 1). The total body computed tomography (CT) scan did not reveal distant metastases; in the left lung, a fibrotic cavity was identified. The disease was staged T3NOM0 according to the American Joint Committee on Cancer 2011 criteria. Laryngectomy was recommended, but the patient refused surgery; therefore, he was referred for definitive radiotherapy. Concomitant chemoradiotherapy was excluded to minimize the risk of more acute complications considering the autoimmune vasculitis, although this disease was in clinical and serological remission at that time. Radiotherapy consisted of a 3D conformal multiportal technique with multileaf collimator-customized 6-10 MV photon beams. The planning target volume included the larynx and neck lymph nodes (bilateral levels II-III-IV) treated to a total dose of $50 \mathrm{~Gy}$ at $2 \mathrm{~Gy} / \mathrm{fr}$, followed by a boost on the larynx to a 70 Gy total prescribed dose delivered over 7 weeks. During the second week of treatment, the patient started to complain of worsening dysphagia and weight loss, recurrent febrile episodes and otalgia. The diagnosis was bilateral otitis media and mild mucositis of the soft palate, and antibiotics with steroids were prescribed. A laryngoscopy revealed a mild mucositis with an initial regression of the visible glottic cancer with stagnant saliva in the pyriform sinuses. Radiotherapy was temporarily discontinued at 30 Gy delivered dose. A few days later the patient developed prolonged febrile episodes (temperature of $39^{\circ} \mathrm{C}$ ); the weight loss worsened, so he was admitted to the hospital. An X-ray and CT scan of the chest revealed pneumonia in the right lung, and the blood tests revealed leukocytosis (WBC count $9,000 / \mathrm{mm}^{3}$ ) with $80 \%$ neutrophils and $18 \%$ lymphocytes. Blood chemistry results included hypogammaglobulinemia ( $8 \%$ of total, $3 \mathrm{~g} / \mathrm{ml}$ ), ferritin $512 \mathrm{ng} / \mathrm{ml}$ (range 22-322 ng/ml), increased erythrocyte sedimentation rate to $100 \mathrm{~mm} / \mathrm{h}$ (normal value $<21 \mathrm{~mm} / \mathrm{h}$ ) and CRP $40 \mathrm{ml} / 1$. Haemofilus influenzae was found in the sputum. Steroids, antifungal and antibiotic therapy were effective and, after 1 week, the CT scan of the chest revealed partial resolution of the pneumonia and radiotherapy was resumed.
A weight loss of $>10 \mathrm{~kg}$ during the following 3 weeks and a progressive lack of appetite with poor oral intake warranted administration of parenteral nutrition via central venous access and nutritional hypercaloric oral supplementation, but no clinical benefit was observed. The dysphagia persisted, and a percutaneous endoscopic gastrectomy (PEG) was ultimately required. At 50 Gy delivered dose the patient underwent laryngoscopy, which revealed fixation of the right hemilarynx, mucosal oedema and a fibrinous secretion on the epiglottis. The swallowing test revealed loss of the oropharyngeal reflex due to IX nerve impairment; in addition, the palpatory elevation of the larynx was absent due to X and XI nerve impairment. The methylene blue test revealed loss of the cough reflex; the swallowing test for semisolid food revealed liquid passing through the larynx and the first tracheal rings. Palsy of the cranial nerves IX, X and XI was diagnosed, and enteral nutrition through PEG was deemed mandatory. The cough reflex was not evocable, and diaphragmatic impairment was observed, requiring tracheostomy. Radiotherapy was again interrupted at 60 Gy delivered dose. An MRI scan of the larynx was performed 1 month later, which revealed oedema of the glottis with shrinkage of the initial cancer (Fig. 2), confirmed by fibroscopic biopsy. The brain MRI with contrast enhancement performed at the same time revealed sinusitis with concentric mucosal thickening of the bilateral sphenoidal, ethmoidal and right maxillary sinuses, with stagnant secretions (Fig. 3), and a focal meningeal thickening of the skull base extending to the mastoid processes below the jugular foramina (Fig. 4). The blood count revealed leukocytosis (WBC 13,000/ $\mathrm{mm}^{3}$ ) with absolute neutrophilia and severe lymphopenia (14\%). Blood biochemistry revealed severe hypogammaglobulinemia (3.24\% of total). The c-ANCA titre was $0.1 \mathrm{UR} / \mathrm{ml}$. The immunophenotype of the lymphocytes involved complete disappearance of the B-lymphocyte series; in particular, the value of $\mathrm{CD} 19^{+} / \mathrm{CD} 20^{+}$lymphocytes was zero. Three months after radiotherapy was discontinued, the patient developed aspiration pneumonia and later an intestinal perforation in the PEG site. Finally, the patient succumbed at the end of April 2012 to fatal complications of progressive ANCA-negative WG, including peritonitis with ascites and thrombosis of the portal veins.

\section{Discussion}

WG is a rare autoimmune disease defined as a systemic vasculitis affecting small- and medium-sized vessels characterized by inflammatory granulomas with necrotizing vasculitis (1). This disease is usually associated with the presence of c-ANCA, which is an established diagnostic marker of WG (9). ANCA-associated vasculitides affect both sexes equally, with a mean age at diagnosis in the fifth decade of life. Almost 93-98\% of the patients are Caucasian or Hispanic, although recent studies have reported an incidence in Japan similar to that in the United Kingdom (10). The estimated annual incidence varies from 2 to 12 cases per million population, with a prevalence of 23-160 cases per million population (11). According to the Chapel Hill consensus conference in 2012, this disease has been included in the group of small-vessel vasculitides as an ANCA-associated vasculitis, and was renamed as GPA (12). As all ANCA-associated 


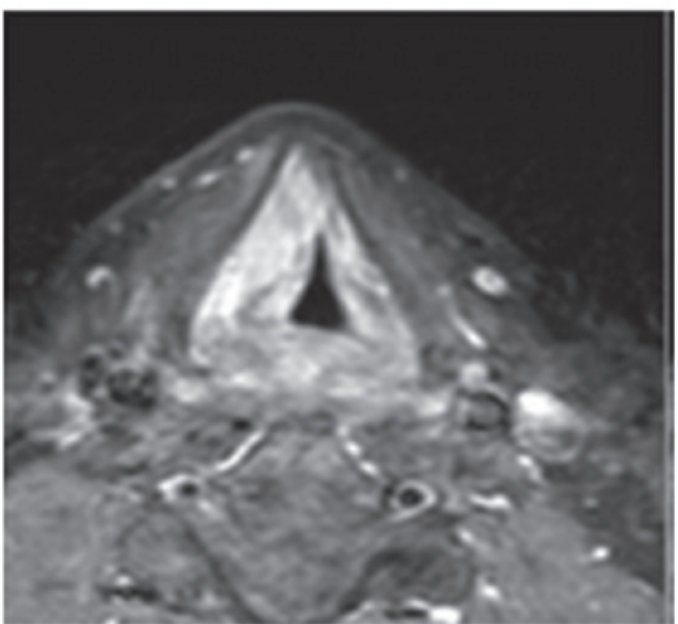

Figure 1. Magnetic resonance T2-weighted axial image of the glottic cancer at diagnosis showing glottic-subglottic stenosis with the tumor involving mainly the right vocal fold.

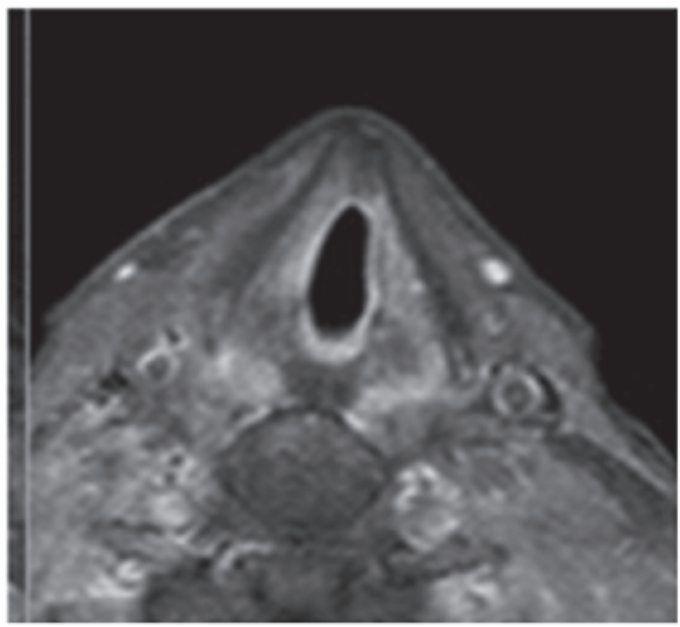

Figure 2. Magnetic resonance T2-weighted axial image demonstrating complete resolution of the glottic cancer after irradiation with $60 \mathrm{~Gy}, 1$ month after interruption of radiotherapy.

vasculitides, GPA is a potentially life-threatening disease, depending on the severity of the clinical manifestations. GPA predominantly affects the upper and lower airways and the kidneys, but other organs may be affected as well, such as the skin, CNS, eyeballs (often presenting with proptosis), and ears (presenting with otitis with progressive hearing loss). The systemic vasculitis form may be lethal when renal or pulmonary involvement leads to alveolar hemorrhage-related respiratory failure or necrotizing glomerulonephritis (13). The patient in the present case had a chronic disease lasting for $>10$ years, with alternating relapses and remissions, with initial respiratory involvement starting from the lung and spreading to the paranasal sinuses. In GPA, the upper respiratory tract symptoms, including rhinosinusitis or otitis media, are the most frequent initial presentations, with a prevalence of $>75 \%$ (13). Moreover, the patient had been heavily treated with the all immunosuppressive therapies available until 2011, including steroids, methotrexate, CYC and RTX. The latter was administered outside clinical trials as an off-label modality for

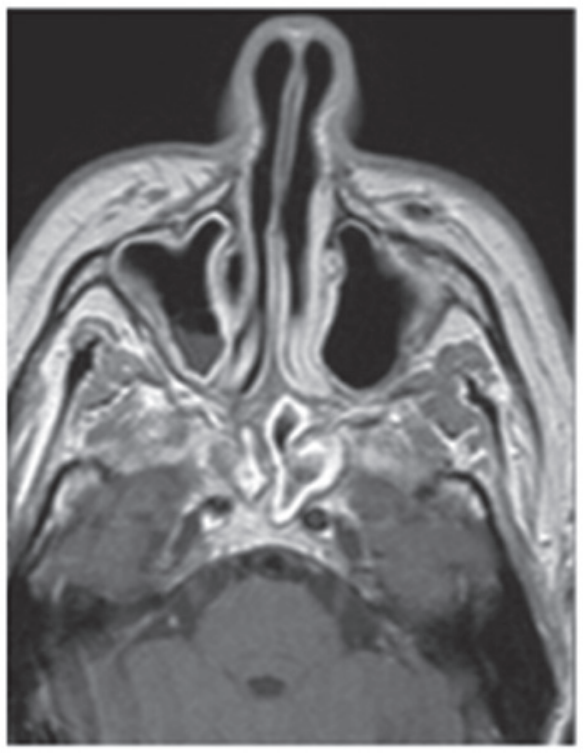

Figure 3. Magnetic resonance T2-weighted axial images of the skull, showing pachymeningitis in the jugular foramina.

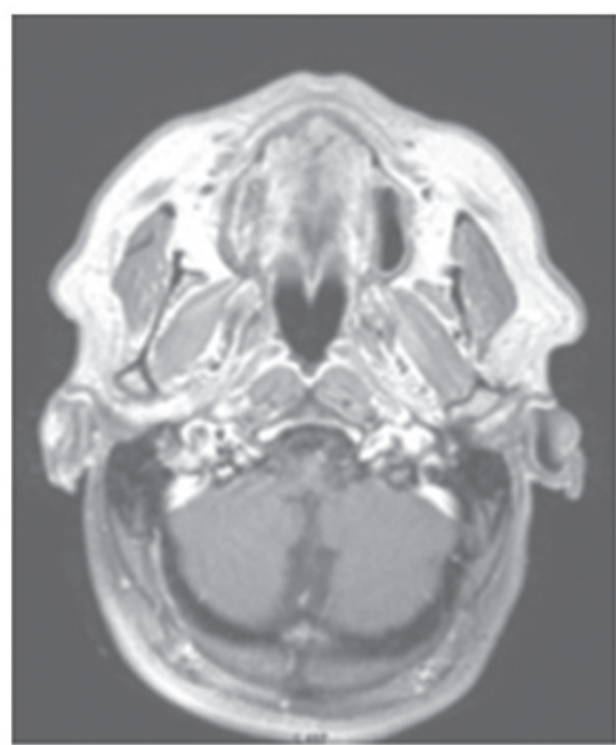

Figure 4. Magnetic resonance T2-weighted axial images of the skull showing sinus involvement.

5 years, until the diagnosis of metachronous glottic cancer. It is unclear whether immunosuppressive therapy or the granulomatosis per se were responsible for cancer development. The patient was admitted to our institution with T3N0 glottic SCC to receive definitive radiotherapy, as surgery had been rejected. The testing for the gag reflex was normal and the patient did not complain of systemic or local symptoms at the beginning of radiation therapy. After delivery of $30 \mathrm{~Gy}$ within the first 3 weeks of treatment, the patient developed clinical symptoms, such as fever and inexplicable weight loss, which are described in 70-100\% of WG patients as the main initial symptoms (13); in the present case, there appeared to be no association with the radiation treatment, as the delivered dose was too low to explain the progressive weight loss. The involuntary weight loss during head and neck cancer treatment 
is a foreseeable event that may have different explanations, such as tumor obstruction with swallowing difficulties, metabolic alterations that may affect appetite, and tissue injury in the irradiated area affecting the ability to eat (14). It has been reported that the weight loss usually appears during the third week of radiotherapy, but becomes most prominent towards the end of treatment in patients treated with concomitant chemoradiotherapy for head and neck cancer (15). Our patient had been treated with radiotherapy alone, and complained of early progressive dysphagia and marked weight loss during the 3rd week of treatment; further specific tests revealed a swallowing impairment due to lower cranial nerve palsies. These findings appeared too early to be attributed to the radiation treatment; in addition, timing and dosage were not supportive of this theory. However, radiation-induced lower cranial nerve palsies (RINCP) may be hypothesized in the present case, despite RINCP having been reported as a long-term complication that appears more frequently during treatment of nasopharyngeal carcinoma as a consequence of radionecrosis of the temporal lobes, or a radiation dose to the cranial nerves originating from the brain stem of >54 Gy (16). In the present case, neurological symptoms occurred at $30 \mathrm{~Gy}$ delivered dose as an acute effect; moreover, after a revision of the treatment plan, the maximum dose to the brain stem at the origin of the cranial nerves was assessed, and was found to be $<50$ Gy.

Cranial nerve palsy due to CNS involvement by WG may be hypothesized. The frequency of CNS involvement according to various studies is $4-11 \%$ of WG cases (13). Dura mater infiltrates, cranial nerve pathology and vasculitis are the most frequent CNS lesions associated with clinical symptoms involving the cranial nerves, such as paresthesia or motor function impairment (17). Our patient developed palsies of the IX, $\mathrm{X}$ and XI cranial nerves originating from the bulbar brain stem and crossing the neck through the jugular foramina (18). Lower cranial nerve palsies are reported as an unusual occurrence, most commonly occurring in the later stages of the disease, with unilateral distribution or associated with pachymeningitis of the skull base (19). Severe dysphagia secondary to paralysis of the lower cranial nerves and phrenic nerve involvement followed by respiratory failure are described during the course of this disease. The most possible cause of cranial nerve palsies in our patient may be a direct extension of the granulomatosis into the jugular foramina, possibly from the middle ear or paranasal sinuses (20), resulting in mechanical compression of the cranial nerves as documented by MRI. As regards the role of c-ANCA in this patient, it remains uncertain, as originally this patient had c-ANCA-positive disease, but the c-ANCA titre was not detectable in the relapsed phase. These antibodies are an established diagnostic tool for WG, more easily used compared with biopsy (21), and are known to be a pathogenic factor in inflammatory processes that underlie necrotizing vasculitis (22). Although the sensitivity of c-ANCA in active WG is up to $91 \%$, with a specificity of $99 \%$ (13), their prognostic role as a marker of the disease remains unclear. Some authors have reported a non-uniform response of the c-ANCA titre after RTX treatment; remission with elevated c-ANCA titre or relapses with undetectable c-ANCA have been recorded $(23,24)$. In the present case, the c-ANCA negativity may be attributed to the past immunotherapies and the steroid administration during radiation treatment (25). In fact, after a long course of immunosuppressive therapies, such as CYC and RTX, the c-ANCA titre may not be detectable due to the consequent hypogammaglobulinemia and lymphopenia $(26,27)$. In our patient, the c-ANCA titre was not detectable, as there was severe hypogammaglobulinemia and lymphopenia, and no mature B lymphocytes $\left(\mathrm{CD} 19^{+} / \mathrm{CD} 20^{+}\right)$were found. Furthermore, RTX is a chimeric monoclonal antibody against CD20 that depletes B-cells and was found to be effective in inducing and maintaining remission of ANCA-associated vasculitis in randomized controlled trials $(28,29)$, so that it has been approved for the treatment of rheumatoid arthritis and recently for induction therapy of AAV. B lymphocytes are key to the pathogenesis of autoimmune diseases by production of autoantibodies and pro-inflammatory cytokines, and are targeted by RTX (30). As AAV frequently relapses, repeated RTX infusions are used as maintenance treatment. However, as RTX induces a long-lasting depletion of B-cells, it has been associated with an altered B-cell maturation capacity (31). Furthermore, prolonged B-cell depletion may lead to decreased antibody production, as assessed by two retrospective studies in which severe infections and hypogammaglobulinemia were frequent adverse events $(26,27)$. Our patient had been receiving RTX since 2007, albeit with an unconventional schedule as an off-label therapy, developing severe lymphopenia coinciding with radiotherapy and steroid administration. Moreover, it is unclear whether the development of glottic cancer should be considered a consequence of the heavy immunosuppressive therapy or a late manifestation of WG. In fact, there are some reports on rare cases of SCC of the nasal cavity arising in patients with GPA $(32,33)$, and there is evidence supporting a high risk of cancer in these patient due to immunosuppression, as recorded by the long-term post-trial follow-up of the WGET cohort (34). Whether radiotherapy may also exert an effect remains controversial. The role of radiotherapy and chemoradiotherapy on the local control, survival and organ sparing $(35,36)$ in laryngeal cancer is well-known. A diagnosis of collagen vascular disease as GPA may predispose to high-grade radiation-induced toxicity, although it has been demonstrated by a case control study that radiotherapy is generally well-tolerated, but bears a higher risk of severe late toxicity when delivered to the pelvis, as in the setting of systemic lupus erythematosus or scleroderma (37). It may be argued that radiotherapy was not effective in this patient, but some studies reported its efficacy in selected patients with solitary WG lesions refractory to systemic therapy or involving critical organs. For example, subglottic stenosis due to fibrosis in a WG patient who was treated with effective low-dose radiation was described in a previous case report (38). Moreover, solitary granulomatosis lesions have successfully resolved with low-dose radiotherapy, as reported in another case report (39). In our patient, 60 Gy delivered radiation dose was effective in reducing the cancer-related glottic stenosis, as shown by the MRI sequences and confirmed by biopsy, confirming its therapeutic effect (Fig. 2).

In conclusion, we herein described a case of glottic cancer in a WG patient treated with radiotherapy alone. The patient developed a fatal flare of the autoimmune disease soon after RTX therapy interruption. The main finding was a c-ANCA-negative relapse with lower cranial nerve palsies, affecting the swallowing 
ability and leading to a marked weight loss during the radiation treatment. Therefore, radiation treatment should be administered with caution to patients affected by AAV who are in remission after immunosuppressive therapy, as the swallowing function may be compromised by acute radiation-related injury and neurological complications may appear as a consequence of the relapse of this collagen vascular disease.

\section{Acknowledgements}

Not applicable.

\section{Funding}

No funding was received.

\section{Availability of data and materials}

The datasets used and/or analyzed during the current study are available from the corresponding author on reasonable request.

\section{Authors' contributions}

GL drafted the manuscript and administered radiotherapy to this patient. ABV provided and revised the MRI images. MADC conducted the swallowing tests and fibroscopic examination. GB managed the medical therapy to this patient. GS contributed to the critical review and supervised the entire work. All authors have read and approved the final version of this manuscript.

\section{Ethics approval and consent to participate}

Not applicable.

\section{Patient consent for publication}

Written informed consent was obtained from the patient for publication of this case report and any accompanying images.

\section{Competing interests}

The authors declare that they have no competing interests to disclose.

\section{References}

1. Bacon PA: The spectrum of Wegener's granulomatosis and disease relapse. N Engl J Med 352: 330-332, 2005.

2. Kim SH, Park J, Bae JH, Cho MS, Park KD and Jeong JH: ANCA-negative Wegener's granulomatosis with multiple lower cranial nerve palsies. J Korean Med Sci 28: 1690-1696, 2013.

3. Armani M, Spinazzi M, Andrigo C, Fassina A, Mantovan M and Tavolato B: Severe dysphagia in lower cranial nerve involvement as the initial symptom of Wegener's granulomatosis. J Neurol Sci 263: 187-190, 2007.

4. Fauci AS and Wolff SM: Wegener's granulomatosis: Studies in eighteen patients and a review of the literature. Medicine (Baltimore) 52: 535-561, 1973.

5. Smith RM, Jones RB and Jayne DR: Progress in treatment of ANCA-associated vasculitis. Arthritis Res Ther 14: 210, 2012.

6. Venhoff N, Effelsberg NM, Salzer U, Warnatz K, Peter HH, Lebrecht D, Schlesier M, Voll RE and Thiel J: Impact of rituximab on immunoglobulin concentrations and B cell numbers after cyclophosphamide treatment in patients with ANCA-associated vasculitides. PLoS One 7: e37626, 2012.
7. Pankhurst T, Savage CO, Gordon C and Harper L: Malignancy is increased in ANCA-associated vasculitis. Rheumatology (Oxford) 43: 1532-1535, 2004.

8. Faurschou M, Sorensen IJ, Mellemkjaer L, Loft AG, Thomsen BS, Tvede N and Baslund B: Malignancies in Wegener's granulomatosis: Incidence and relation to cyclophosphamide therapy in a cohort of 293 patients. J Rheumatol 35: 100-105, 2008.

9. Russell KA, Fass DN, Speks U. Clinical and prognostic value of antineutrophil cytoplasmic antibodies in Wegener's granulomatosis and microscopic polyangiitis : Comment on the article by Russell et al. Arthritis and Rheumatology 46: 278-280, 2002.

10. Fujimoto S, Watts RA, Kobayashi S, Suzuki K, Jayne DR, Scott DG, Hashimoto H and Nunoi H: Comparison of the epidemiology of anti-neutrophil cytoplasmic antibody-associated vasculitis between Japan and U.K. Rheumatology (Oxford) 50: 1916-1920, 2011.

11. Mohammad AJ, Jacobsson LT, Westman KW, Sturfelt G and Segelmark M: Incidence and survival rates in Wegener's granulomatosis, microscopic polyangiitis, Churg-Strauss syndrome and polyarteritis nodosa. Rheumatology (Oxford) 48: 1560-1565, 2009.

12. Jennette JC, Falk RJ, Bacon PA, Basu N, Cid MC, Ferrario F, Flores-Suarez LF, Gross WL, Guillevin L and Hagen EC: 2012 revised International Chepel Hill Consensus Conference Nomenclature of Vasculitides. Arthritis Rheum 65: 1-11, 2013.

13. Pagnoux C: Updates in ANCA-associated vasculitis. Eur J Rheumatol 3: 122-133, 2016.

14. Capuano G, Grosso A, Gentile PC, Battista M, Bianciardi F, Di Palma A, Pavese I, Satta F, Tosti M, Palladino A, et al: Influence of weight loss on outcomes in patients with head and neck cancer undergoing concomitant chemoradiotherapy. Head Neck 30: 503-508, 2008.

15. Paccagnella A,Morello M, Da Mosto MC, Baruffi C, Marcon ML, Gava A, Baggio V, Lamon S, Babare R, Rosti G, et al: Early nutritional intervention improves treatment tolerance and outcomes in head and neck cancer patients undergoing concurrent chemoradiotherapy. Support Care Cancer 18: 837-845, 2010.

16. Luk YS, Shum JSF, Sze HCK, Chan LL, Ng WT and Lee AW: Predictive factors and radiological features of radiation-induced cranial nerve palsy in patients with nasopharyngeal carcinoma following radical radiotherapy. Oral Oncol 49: 49-54, 2013.

17. Cattaneo L, Chierici E, Pavone L, Grasselli C, Manganelli P, Buzio C and Pavesi G: Peripheral neuropathy in Wegener's granulomatosis, Churg-Strauss syndrome and microscopic polyangiitis. J Neurol Neurosurg Psychiatry 78: 1119-1123, 2007.

18. Chiarugi G and Bucciante L: Istituzioni di Anatomia dell'Uomo, 10th edition. Vallardi, Milan, pp719-743, 1968-1972 (In Italian).

19. Nishino H, Rubino FA, DeRemee RA, Swanson JW and Parisi JE: Neurological involvement in Wegener's granulomatosis: An analysis of 324 consecutive patients at the Mayo Clinic. Ann Neurol 33: 4-9, 1993.

20. Kashiyama T, Suzuki A and Mizuguchi K: Wegener's granulomatosis with multiple cranial nerve involvements as the initial clinical manifestations. Intern Med 34: 1110-1113, 1995.

21. Nölle B, Specks U, Lüdemann J, Rohrbach MS, DeRemee RA and Gross WL: Anticytoplasmic autoantibodies: Their immunodiagnostic value in Wegener granulomatosis. Ann Intern Med 111: 28-40, 1989

22. Finkielman JD, Lee AS, Hummel AM, Viss MA, Jacob GL, Homburger HA, Peikert T, Hoffman GS, Merkel PA, Spiera R, et al: WGET Research Group: ANCA are detectable in nearly all patients with active severe Wegener's granulomatosis. Am J Med 120: 643.e9-643.e14, 2007.

23. Aries PM, Hellmich B, Voswinkel J, Both M, Nölle B, Holl-Ulrich K, Lamprecht P and Gross WL: Lack of efficacy of rituximab in Wegener's granulomatosis with refractory granulomatous manifestations. Ann Rheum Dis 65: 853-858, 2006.

24. Omdal R, Wildhagen K, Hansen T, Gunnarsson R and Kristoffersen G: Anti-CD20 therapy of treatment-resistant Wegener's granulomatosis: Favourable but temporary response. Scand J Rheumatol 34: 229-232, 2005.

25. Hebert LA, Ardoin S and Shim RL: Rituximab or cyclophosphamide in ANCA-associated renal vasculitis. N Engl J Med 363: 2073-2074, 2010

26. Besada E, Koldingsnes W and Nossent JC: Serum immunoglobulin levels and risk factors for hypogammaglobulinaemia during long-term maintenance therapy with rituximab in patients with granulomatosis with polyangiitis. Rheumatology (Oxford) 53: 1818-1824, 2014. 
27. Jones RB, Ferraro AJ, Chaudhry AN, Brogan P, Salama AD, Smith KGC, Savage CO and Jayne DR: A multicenter survey of rituximab therapy for refractory antineutrophil cytoplasmic antibody-associated vasculitis. Arthritis Rheum 60: 2156-2168, 2009.

28. Jones RB, Tervaert JW, Hauser T, Luqmani R, Morgan MD, Peh CA, Savage CO, Segelmark M, Tesar V, van Paassen P, et al; European vasculitis study group: Rituximab versus cyclophosphamide in ANCA-associated renal vasculitis. N Engl J Med 363: 211-220, 2010

29. Stone JH, Merkel PA, Spiera R, Seo P, Langford CA, Hoffman GS, Kallenberg CG, St Clair EW, Turkiewicz A, Tchao NK, et al; RAVE-ITN Research Group: Rituximab versus cyclophosphamide for ANCA-associated vasculitis. N Engl J Med 363: 221-232, 2010

30. Looney RJ: B cells as a therapeutic target in autoimmune diseases other than rheumatoid arthritis. Rheumatology (Oxford) 44 (Suppl 2): ii13-ii17, 2005.

31. Thiel J, Rizzi M, Engesser M, Dufner AK, Troilo A, Lorenzetti R, Voll RE and Venhoff N: B cell repopulation kinetics after rituximab treatment in ANCA-associated vasculitides compared to rheumatoid arthritis, and connective tissue diseases: A longitudinal observational study on 120 patients. Arthritis Res Ther 19: 101, 2017.

32. Stein J, Sridharan ST, Eliachar I, Niv A, Wood B and Hoffman GS: Nasal cavity squamous cell carcinoma in Wegener's granulomatosis. Arch Otolaryngol Head Neck Surg 127: 709-713, 2001.

33. Kuan EC, Peng KA, Gonzales LO and Sercarz JA: A case of squamous cell carcinoma of the nasal cavity in a patient with granulomatosis with polyangiitis (Wegener granulomatosis). Ear Nose Throat J 97: E37-E41, 2018.
34. Silva F, Seo P, Schroeder D, Stone JH, Merkel PA, Hoffman GS, Spiera R, Sebastian JK, Davis JC Jr, St Clair EW, et al: Solid malignancies among patients with Wegener's granulomatosis treated with Etarnercept: Long-term follow-up of a multicenter longitudinal cohort. Arthritis Rheum 63: 2495-2503, 2011.

35. Pignon JP, le Maître A, Maillard E and Bourhis J; MACH-NC Collaborative Group: Meta-analysis of chemotherapy in head and neck cancer (MACH-NC): An update on 93 randomised trials and 17,346 patients. Radiother Oncol 92: 4-14, 2009.

36. Forastiere AA, Zhang Q, Weber RS, Maor MH, Goepfert H, Pajak TF, Morrison W, Glisson B, Trotti A, Ridge JA, et al: Long-term results of RTOG 91-11: A comparison of three nonsurgical treatment strategies to preserve the larynx in patients with locally advanced larynx cancer. J Clin Oncol 31: 845-852, 2013.

37. Lin A, Abu-Isa E, Griffith KA and Ben-Josef E: Toxicity of radiotherapy in patients with collagen vascular disease. Cancer 113: 648-653, 2008

38. Neviani CB, Carvalho HA, Hossamu C, Aisen S and Nadalin W: Radiation therapy as an option for upper airway obstruction due to Wegener's granulomatosis. Otolaryngol Head Neck Surg 126 195-196, 2002.

39. Wygoda A, Rutkowski T, Składowski K and Hejduk B: Low dose radiotherapy as an effective treatment in a patient with solitary Wegener's granulomatosis resistant to systemic treatment - case report. Contemp Oncol (Pozn) 17: 107-111, 2013. 